\title{
Modeling and Optimization of Coal Moisture Control System Based on BFO
}

\author{
Xiaobin Li \\ School of Electrical and Electronic Engineering \\ Shanghai Institute of Technology \\ Shanghai P.R. China \\ e-mail: xbli@sit.edu.cn \\ Yang Yu \\ School of Electrical and Electronic Engineering \\ Shanghai Institute of Technology \\ Shanghai P.R. China \\ e-mail: 704403329@qq.com
}

\author{
Ting $\mathrm{Hu}$ \\ School of Electrical and Electronic Engineering \\ Shanghai Institute of Technology \\ Shanghai P.R. China \\ e-mail: estella_genna@yahoo.cn \\ Haiyan Sun \\ School of Ecological Technology and Engineering \\ Shanghai Institute of Technology \\ Shanghai P.R. China \\ e-mail: haiyansun@sit.edu.cn
}

\begin{abstract}
Coal moisture control process is a critical process in energy saving, pollution reduction and improving production efficiency and the quality of coke. To achieve precise control of coal moisture control system, against their strong coupling, large, nonlinear systems with time-delay characteristics using the RBF artificial neural network approach for modeling. And use the bionic BFO (Bacterial Foraging Optimization) according to the fitness to optimize the RBF Neural network parameters. Also compare the RBF Neural network performance optimized by these bionic BFO in order to achieve better results. This method provides a theoretical basis for accurate control of coal moisture process. To created the conditions for the reduction of energy and pollution with improving the quality of coke.
\end{abstract}

Keywords- coal moisture control; BFO; modeling; optimization

\section{INTRODUCTION}

CMC (coal moisture control) is a key procedure in coking to save energy, reduce pollution and improve productivity and quality. It is through direct or indirect heating that coal moisture is controlled within about $6 \%$ before putting into furnace and remain stable to ensure production with high efficiency and low consumption.

Coal moisture control system is a multi-parameter, strong coupling and non-linear system with large time delay, of which the model can hardly be established through mechanism. Online moisture monitoring on the dryer gate rarely provides reliable data for the disturbance of moisture and dust. In production, there is about 15 hour delay in detecting the moisture of coal out of the dryer and provides little reference to real production. Such large time delay can be handled when the moisture is stable, but will influence the effect of moisture control system when the coal moisture out of the dryer is quite sensitive to the weather in open-air coal ground in rainy season in southern China. Therefore, it is of great importance to find relationships between such parameters as steam consumption and flue gas temperature, moisture of coal in and out of the dryer under different conditions by analyzing and thus establishing control system to realize automatic online control of coal moisture.

For the modeling of coal moisture control, Ergun ${ }^{[1]}$ adopts linear partial differential equation to describe the mathematical model of coal drying in prompt dropping phase assuming that evaporation only occurs in the surface of solid, which is not satisfactory to coal with complicated construction. For the strong coupling of coal moisture control, Gao Jianjun ${ }^{[2]}$ provides a mathematical model based on equilibrium between moisture and coal and thermal balance of control volume with moisture control reactor as the control volume, to calculate the flue gas volume, temperature of in and out waste gas and effect of coal moisture before treating on the humidified coal powder and waste gas moisture. The study adopts Bacterial Foraging Optimization (BFO) algorithm to establish and optimize the RBF Neural Network Model based on thermal equilibrium theory and relevant analysis so as to solve the problems occurs in other modeling.

\section{PROCESS AND MODELING}

\section{A. Process}

Coal moisture controlling system in Bao Steel is studied and indirect type steam tube rotary dryer, dust catcher, fluid reservoir, flash tank, draught fan, pipeline, steam tube and conveyer belt and etc., are involved in the system. Figure 1 shows its process.

In the process, there are over 30 factors that influence directly or indirectly the coal moisture after drying, including output, stream pressure, pressure of condensate tank, steam temperature, real steam flow, steam pipeline flow, main motor current, current of feed screw and discharge screw, current of draught fan, output of draught fan, CO strength in flue gas, steam flow heated by recycle gas, temperature of recycle gas, outlet gas flow rate, recycle gas flow rate, blast volume, pressure of dryer inlet, temperature of blast, pressure differences of dust catcher, gas temperature of dryer 
outlet, gas temperature of inset and outlet, gas pressure of inlet and outlet, bag temperature of catcher, dust hopper temperature, temperature and pressure of reverse nitrogen, oxygen content of desiccant and inlet coal moisture.

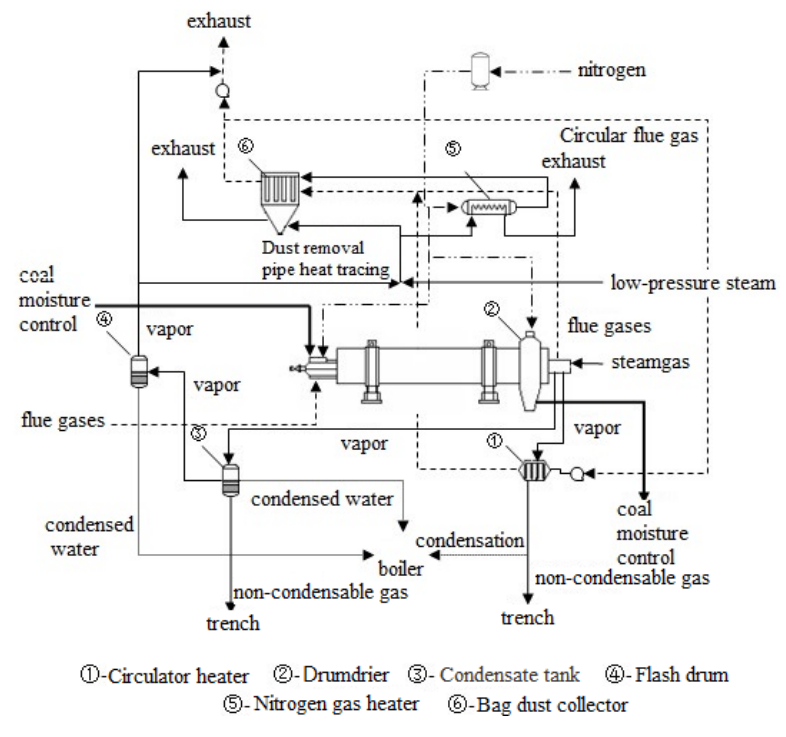

Fig.1 Coal Moisture Control process

By analyzing the data of coal moisture controlling based on thermal equilibrium mechanism and mathematical statistics theory and relationships between variables with the aim of saving energy and reducing consumption with high control accuracy, such independent variables most relevant to steam volume are identified to be as input and output of the control system, including coal to be treated, gas temperature of dryer outlet, inlet coal moisture, outlet coal moisture.

\section{B. Modeling}

For the characteristics of non-linear, multi-input and strong coupling and low accuracy of online detecting of outlet coal moisture, RBF artificial neural network is adopted for modeling. Figure 2 shows the structure, of which $\mathrm{X}_{1}, \mathrm{X}_{2} \ldots$ are inputs, $\mathrm{C}_{1}, \mathrm{C}_{2}, \mathrm{C}_{\mathrm{k}}$ are center vectors, $\Phi\left(\left\|x-c_{k}\right\|, s_{k}\right)$ is Gauss function, $w_{k i}$ is weight

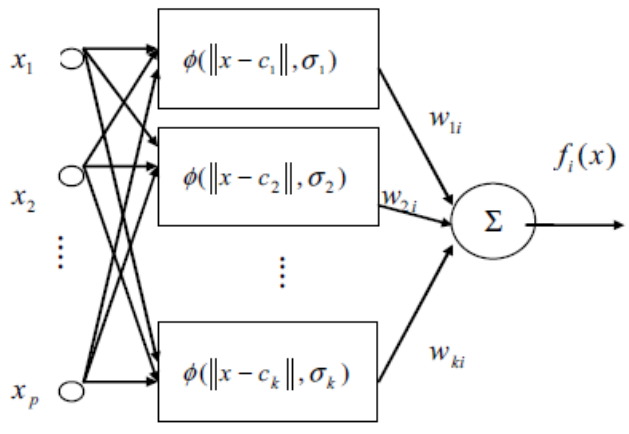

Fig.2 RBF Artificial neural network
Number of units in input layer: as the input layer is the cache of outer data and depends on the dimensions of input vectors, the number of units in input layer is set as 3 .

Number of units in output layer: to simplify the system and save the cost of calculation, the number of units in output layer is set as 1 . data.

Number of hidden units: is set as 8 after training 100 set

\section{DATA ACQUISITION}

The parameters of environment, operation and working of coal moisture controlling system and the period of coal in the rotary kiln are recorded in the field and sample coal is taken back to lab. Figure 3 shows the monitoring process.

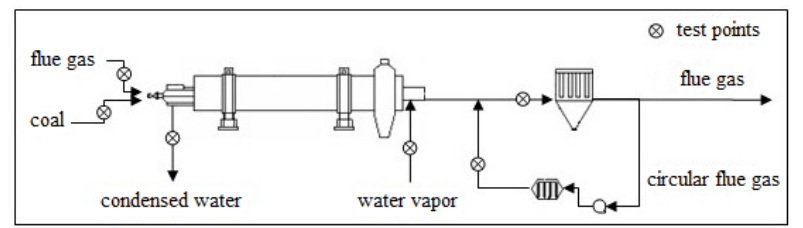

Fig.3 Testing Points of the System

720 set of data is recoded at the same time lap by workers of 4 round working time in Bao Steel work shop, of which 310 set is chosen as training samples. Seen as in Table 1.

TAB. I TRAINING SAMPLES

\begin{tabular}{lllll}
\hline $\begin{array}{l}\text { output } \\
\text { (ton/h) }\end{array}$ & $\begin{array}{l}\text { Outlet } \\
\text { temperature of } \\
\text { dryer } \\
\text { (centigrade) }\end{array}$ & $\begin{array}{l}\text { Inlet } \\
\text { moisture } \\
(\% \mathrm{RH})\end{array}$ & $\begin{array}{l}\text { coal } \\
\text { rate } \\
(\mathrm{kg} / \mathrm{h})\end{array}$ & $\begin{array}{l}\text { Steam } \\
\text { flow }\end{array}$ \\
\hline 247.9291 & 74.9978 & 12.1207 & 11088 \\
251.2817 & 70.5444 & 12.0825 & 10939 & \\
247.2381 & 81.2406 & 12.1175 & 11043 & 10948 \\
249.4915 & 76.2792 & 12.0942 & 11510 & \\
243.3044 & 81.7253 & 12.2356 & 10829 & \\
252.8835 & 77.3085 & 12.0127 & 11146 \\
247.4501 & 81.5356 & 12.1405 & 11768 \\
247.0261 & 81.6410 & 12.1640 & 11142 & \\
249.7035 & 81.5356 & 12.1405 & 11502 & \\
248.5415 & 81.5778 & 12.1399 & 11888 \\
245.2909 & 81.5988 & 12.2353 & $\ldots$ \\
244.1760 & 79.8083 & 12.2839 & \\
248.0547 & 81.0720 & 12.1752 & \\
$\ldots$ & $\ldots$ & $\ldots$ & & \\
\hline
\end{tabular}

\section{PARAMETERS IDENTIFICATION AND OPTIMIZATION}

Bacteria Foraging Optimization (BFO) is adopted for parameters identification and optimization of the model. First proposed by Passino in 2002, BFO is a newly developed nature-inspired algorism based on the foraging movement of $E$ coli with the advantages of low sensitivity to initial value and parameters, good robustness, ease to realize and parallel nature and overall searching.

\section{A. BFO Algorism}

Four principal mechanisms are usually included in a BFO: chemotaxis, swarming, reproduction and eliminationdispersal. 
Let us define the movement of coli by the following:

Let $j$ be the index for the chemotactic step, $k$ be the index for the reproduction step, $l$ be the index of the elimination-dispersal event, Also let:

$P$ : Dimension of the search space,

$S$ : Total number of bacteria in the population,

$N_{c}$ : The number of chemotactic steps,

$N_{s}$ : The swimming length,

$N_{r e}$ : The number of reproduction steps,

$N_{e d}$ : The number of elimination-dispersal events,

$P_{e d}$ : Elimination-dispersal probability,

$C(i)$ : The size of the step taken in the random direction specified by the tumble.

- Chemo taxis: Then in computational chemo taxis the movement of the bacterium $i$ may be represented by:

$$
\text { - } \quad \theta^{\mathrm{i}}(j+1, k, l)=\theta^{\mathrm{i}}(j, k, l)+C(i) \frac{\Delta(i)}{\sqrt{\Delta^{T}(i) \Delta(i)}}
$$

- where $\Delta$ indicates a vector in the random direction.

- Swarming: The cell-to-cell signaling in E. coli swarm may be represented by the following function.

$$
\begin{aligned}
& J_{c c}(\theta, P(j, k, l))=\sum_{i=1}^{S} J_{c c}\left(\theta, \theta^{i}(j, k, l)\right) \\
& =\sum_{i=1}^{S}\left[-d_{\text {atrrac tan }} \exp \left(-w_{\text {attrac tant }} \sum_{m=1}^{P}\left(\theta_{m}-\theta_{m}^{i}\right)^{2}\right]\right. \\
& +\sum_{i=1}^{S}\left[h_{\text {repellant }} \exp \left(-w_{\text {repellant }} \sum_{m=1}^{P}\left(\theta_{m}-\theta_{m}^{i}\right)^{2}\right]\right.
\end{aligned}
$$

where $d_{\text {attractant }}$ is the depth of attractant, $w_{\text {attractant }}$ is the width of attractant, $h_{\text {repellant }}$ is the height of repellant, $w_{\text {repellant }}$ is the width of repellant, $\theta^{i}$ is the $m$ th field of bacteria $i, \theta_{m}$ is the $m$ th field of other bacterium in the

- Reproduction: the function is as follows: population.

$$
J_{\text {health }}^{i}=\sum_{j=1}^{N c+1} J(i, j, k, l)
$$

where for the given $k, l$ and $i=1,2, \ldots S$, let $J_{\text {health }}^{i}$ be the health of the bacterium (a measure of how many nutrients it got over its lifetime and how successful it was at avoiding noxious substances). Sort bacteria and chemotactic parameters $C(i)$ in order of ascending cost $J_{\text {health }}^{i}$, and higher cost means lower health. $S_{r}=S / 2$ with the lowest $J_{\text {health }}^{i}$ value die, and the remaining $S_{r}$ bacteria with the best values split by the copies that are made are placed at the same location as their parent.

- Swarming: The cell-to-cell signaling in E. coli swarm may be represented by the following function. Step1: Initialize parameters

$$
p, S, N_{c}, N_{s}, N_{r e}, N_{e d}, P_{e d}, C(i)(i=1,2, . ., S), \theta^{i}
$$

Step2: Elimination-dispersal loop: $l=l+1$

Step3: Reproduction loop: $k=k+1$

Step4: Chemotaxis loop: $j=j+1$

(1) For $i=1,2, \ldots S$ take a chemotactic step for bacterium $i$.

(2) Compute fitness function $J(i, j, k, l)$, let

$$
J(i, j, k, l)=J(i, j, k, l)+J_{c c}(\theta i(j, k, l), P(j, k, l))
$$

(i.e. add on the cell-to cell attractant-repellant profile to simulate the swarming behavior)

(3) Let to save this value since we may find a better cost via a run.

$$
J_{\text {last }}=J(i, j, k, l)
$$

(4) Tumble: generate a random vector

$\Delta(i) \in \mathfrak{R}^{P}$ with each element $\Delta_{m}(i),(m=1,2, \ldots p)$ a random number on $[-1,1]$.

(5) Move: Let

$$
\theta^{\mathrm{i}}(j+1, k, l)=\theta^{\mathrm{i}}(j, k, l)+C(i) \frac{\Delta(i)}{\sqrt{\Delta^{T}(i) \Delta(i)}}
$$

This results in a step in the direction of the tumble for bacterium $i$.

(6) Compute $J(i, j+1, k, l)$ and let

$$
\begin{aligned}
& J(i, j+1, k, l)=J(i, j, k, l) \\
& +J_{c c}\left(\theta^{i}(j+1, k, l), P(j+1, k, l)\right)
\end{aligned}
$$

(7) Swim:

Let $m=0$, then $m<N_{s}$

$$
\begin{aligned}
& \text { Let } m=m+l \text {, if } J(i, j+1, k, l)<J_{\text {last }} \\
& \text { Let } J_{\text {last }}=J(i, j+1, k, l) \text {, then } \\
& \qquad \theta^{\mathrm{i}}(j+1, k, l)=\theta^{\mathrm{i}}(j, k, l)+C(i) \frac{\Delta(i)}{\sqrt{\Delta^{T}(i) \Delta(i)}}
\end{aligned}
$$

go back to Step 6 , and recalculate $J(i, j+1, k, l)$ with $\theta^{\mathrm{i}}(j+1, k, l)$, otherwise, let $m=N_{s}$

(8) Go back to Step 2 and go to next bacteria $i+1$

Step5 : if $j<N_{c}$, go back to Step4. In this case continue chemotaxis since the life of the bacteria is not over.

Step6 : Reproduction: for the given $k, l$ and $i=1,2, \ldots . S$, let $J_{\text {health }}^{i}$ be the health of the bacterium (a measure of how many nutrients it got over its lifetime and how successful it was at avoiding noxious substances). Sort bacteria and chemotactic parameters $C(i)$ in order of ascending cost $J_{\text {health}}^{i}$, and higher cost means lower health. $S_{r}=S / 2$ with the lowest $J_{\text {health }}^{i}$ value die, and the remaining $S_{r}$ bacteria with the best values split by the copies that are made are placed at the same location as their parent. 
Step7: If $k<N r e$, to back to Step3。

Step8 : Elimination-dispersal: For $\mathrm{i}=2,1 \ldots, \mathrm{S}$ with probability $P_{e d}$, eliminate and disperse each bacterium (this keeps the number of bacteria in the population constant). To do this, if a bacterium is eliminated, simply disperse another one to a random location on the optimization domain. If $l<N e d$, then go to Step 2; otherwise end.

\section{B. Adaptive BFO Algorism ${ }^{[4]}$ [5]}

In the initial period of searching optimum, large step helps to avoid local optima and find the area where optima is while in the half period, small step helps to improve accuracy.

Evaluate fitness to the step in chemotaxis to adjust step which each bacteria takes.

Step1: Evaluate fitness

$$
V=\frac{J_{i}}{J_{\max }}\left(X_{\max }-X_{\min }\right) * \text { rand }
$$

where $V$ is fitness, $X_{\max } 、 X_{\min }$ are the boundaries of variables and $J$ is fitness.

Step2: Tumble: generate a random direction unit $\Delta(i)$ to optimize steps according to the following function:

$$
\theta^{\mathrm{i}}(j+1, k, l)=\theta^{\mathrm{i}}(j, k, l)+C(i) \frac{\Delta(i)}{\sqrt{\Delta^{T}(i) \Delta(i)}}
$$

to update the location and its corresponding fitness of each bacteria.

Step3: Swim: optimized by the following function:

$$
C(i)=C(i) * V
$$

Step4: Linear gradient fitness is represented by the following fuction:

$$
V=\frac{\text { step }_{\max }-\text { step }_{\min }}{\text { step }_{\max }} * V
$$

\section{Identification and Optimization}

To improve the prediction effect of the model, BFO with step of 0.1 and 0.5 , adaptive BFO algorism are used to optimize RBF network and the following are the procedures:

Step1: Train weight of each unit by RBF algorism;

Step 2: Calculate J=MSE of each network as its fitness;

Step 3: Search optima of fitness $\mathrm{J}$ by 5 algorisms to find the minimum value of which the corresponding weight is the optima of the network. As seen in Figure 4.

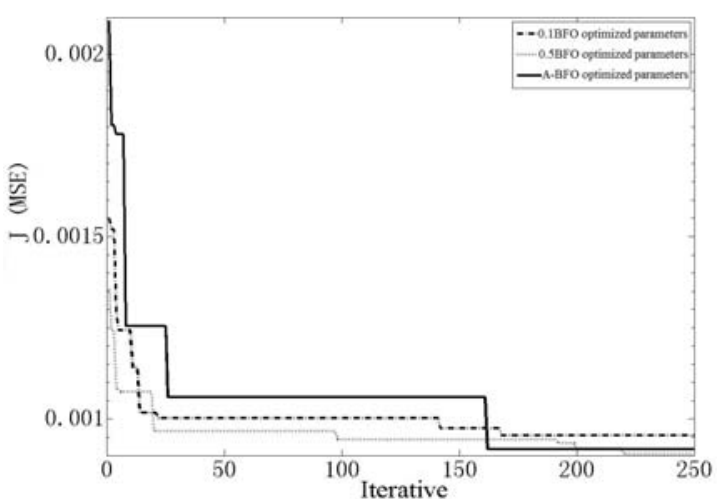

Fig. 4 BFO Optimized RBF Parameters

As shown in Figure 4, when optimized by BFO, the convergence rate of parameters in $\mathrm{RBF}$ is $0.5 \mathrm{BFO}, \mathrm{ABFO}$, $0.1 \mathrm{BFO}$, of which $0.5 \mathrm{BFO}$ is faster.

For the parameters with high dimensions (the RBF network has 40 dimensions), 0.1 BFO leads to local optima.

\section{Model Checking}

From 720 sets data, 310 are chosen in the prediction model.

TAB. II SAMPLES CHECKING

\begin{tabular}{lllll}
\hline $\begin{array}{l}\text { output } \\
\text { (ton/h) }\end{array}$ & $\begin{array}{l}\text { Outlet } \\
\text { temperature } \\
\text { dryer } \\
\text { (centigrade) }\end{array}$ & $\begin{array}{l}\text { Inlet } \\
\text { moisture } \\
(\% R H)\end{array}$ & $\begin{array}{l}\text { coal } \\
\text { rate } \\
(\mathrm{kg} / \mathrm{h})\end{array}$ & $\begin{array}{l}\text { Steam } \\
\text { flow }\end{array}$ \\
\hline 249.7035 & 77.0774 & 12.0613 & 10900 \\
250.6064 & 71.4266 & 12.0960 & 10977 \\
299.3966 & 82.9053 & 10.9383 & 9702 \\
248.1332 & 81.3881 & 12.0948 & 10963 \\
253.0326 & 81.7253 & 11.9765 & 10777 \\
250.9363 & 73.5483 & 12.0954 & 10969 \\
257.5866 & 81.3881 & 11.8725 & 10729 \\
245.5814 & 81.3038 & 12.1985 & 11360 \\
251.5879 & 74.0945 & 12.0960 & 10977 \\
250.4573 & 75.3969 & 12.1302 & 11122 \\
246.6650 & 81.1774 & 12.1991 & 11367 \\
250.8892 & 81.6831 & 12.0831 & 10940 \\
248.5415 & 81.1353 & 12.1558 & 10612.52 \\
$\ldots$ & $\ldots$ & $\ldots$ & $\ldots$ \\
\hline
\end{tabular}

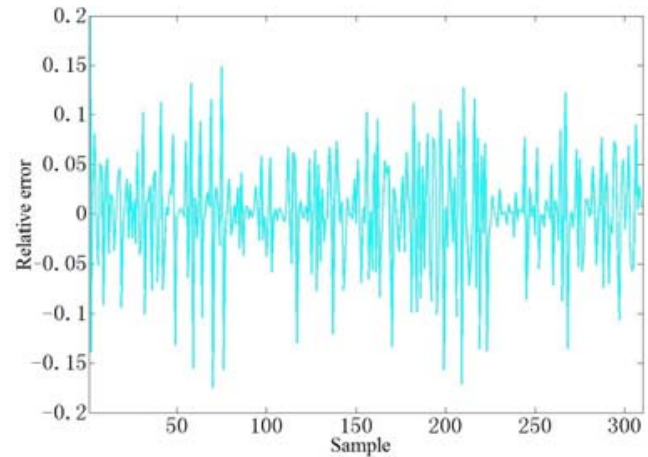

Fig.5 Prediction Difference of RBF without Optimization 


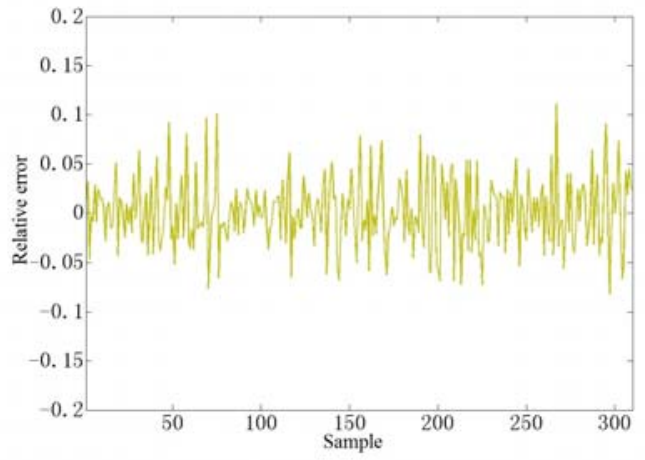

Fig. 6 Prediction Difference of Step 0.1 BFO Optimized RBF Network

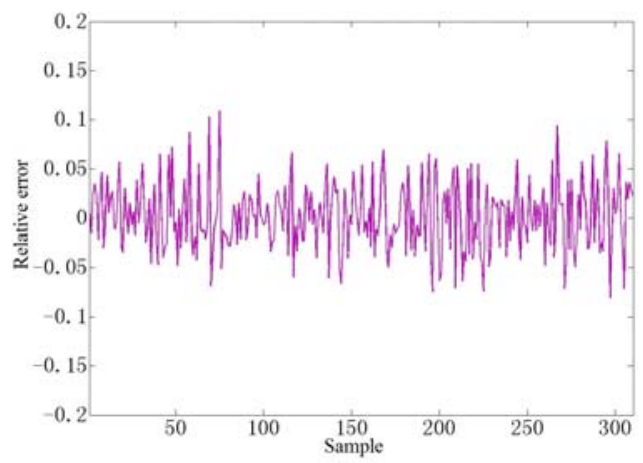

Fig.7 Prediction Difference of ABFO Optimized RBF Network

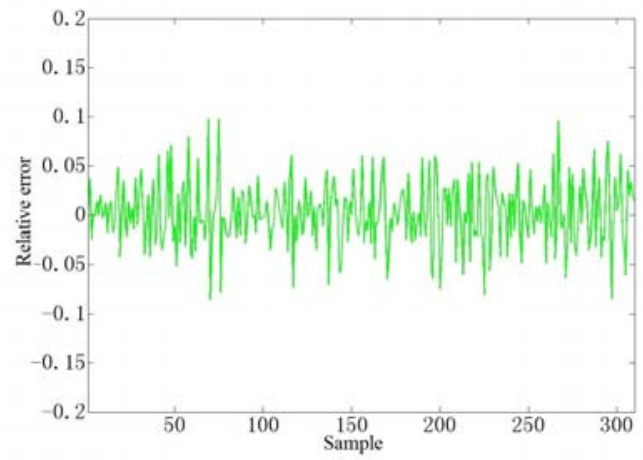

Fig.8 Prediction Difference of Step 0.5 BFO Optimized RBF Network

As Figure 5 to 8 show that the difference of RBF network without optimization reaches as high as $18 \%$ with small difference in only limited areas, which leads to large difference in overall control. The accuracy of $0.5 \mathrm{BFO}$ is high than that of $0.1 \mathrm{BFO}$ ( $0.1 \mathrm{BFO}$ leads to local optima), of which the difference remains within $10 \%$ and $13 \%$ respectively. The difference of ABFO remains within $11 \%$, falling in between of above.

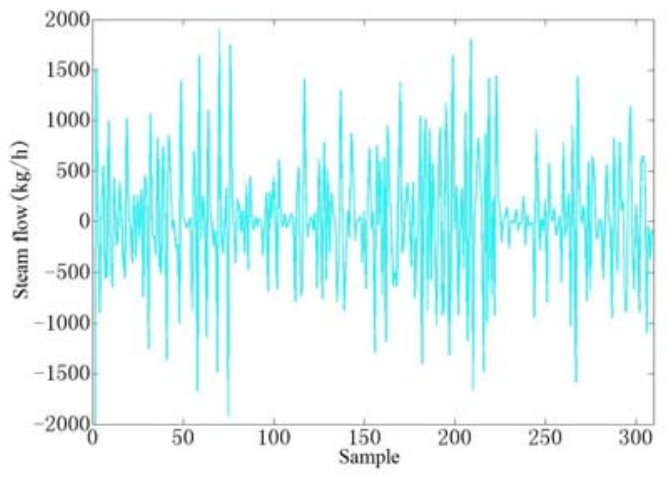

Fig. 9 Difference between Real Value and RBF Prediction Output

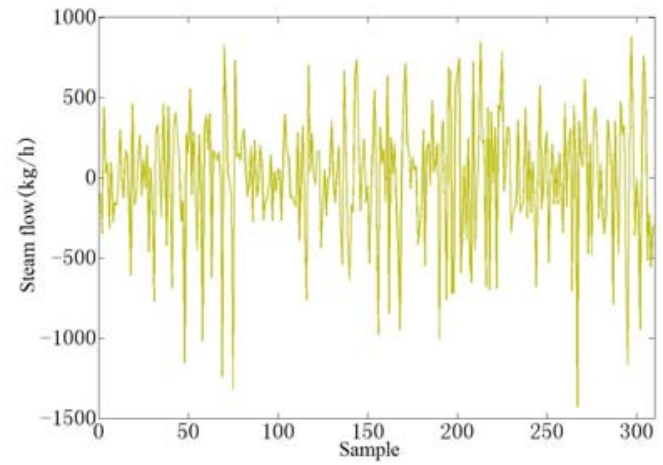

Fig.10 Difference between Real Value and Step 0.1 BFO * RBF Output

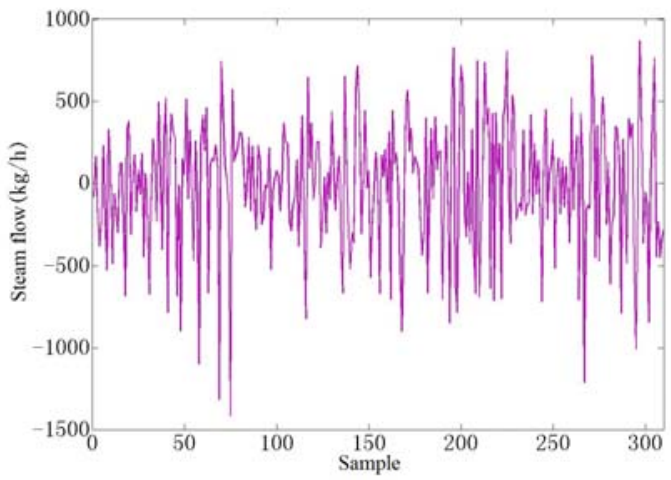

Fig.11 Difference between Real Value and ABFO* RBF Output 


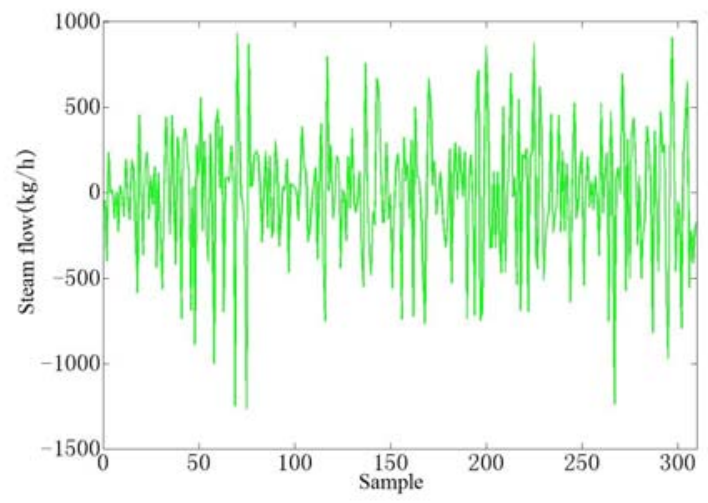

Fig.12 Difference between Real Value and Step 0.5 BFO * RBF Output

Figure 9 to 12 show the difference between real steam flow rate and prediction output of RBF network based on 310 sets data, of which the real steam flow rate is within the range of from $8000 \mathrm{~kg} / \mathrm{h}$ to $20000 \mathrm{~kg} / \mathrm{h}$.

Compared with real steam flow rate, the predicted output of RBF network without optimization fluctuates at as much as $4000 \mathrm{~kg} / \mathrm{h}$ and $0.1 \mathrm{BFO}$ optimized RBF fluctuates at $2300 \mathrm{~kg} / \mathrm{h}$, adaptive $\mathrm{BFO}$ at $2250 \mathrm{~kg} / \mathrm{h}$ and $0.5 \mathrm{BFO}$ one at $2200 \mathrm{~kg} / \mathrm{h}$.

From above, the RBF network optimized by BFO with 0.5 step yields the best result, adaptive BFO falls in between $0.1 \mathrm{BFO}$ and $0.5 \mathrm{BFO}$.

\section{CONCLUSION}

(1) A nature-inspired BFO modeling and optimization of coal moisture controlling system is studied and provided by analyzing its process and existed models.
(2) When optimizing RBF network by BFO, the convergence rate of $\mathrm{BFO}$ with 0.5 step is fastest and followed by ABFO and BFO with 0.1 step.

(3) When identifying RBF network by BFO, the accuracy is $0.5 \mathrm{BFO}, \mathrm{ABFO}$ and $0.1 \mathrm{BFO}$ in sequence, with $0.5 \mathrm{BFO}$ being the highest.

(4) BFO usually leads to local optima to RBF network with high dimensions (the RBF network has 40 dimensions). In the study, RBF network optimized by $0.1 \mathrm{BFO}$ falls in local optima, of which the accuracy is lower than that of 0.5 step BFO.

\section{ACKNOWLEDGMENT}

This work is partially supported by Shanghai key scientific research project No.11510502700, and science and technology innovation focus of SHMEC No.12ZZ189, andScience Foundation of SIT (YJ2011-33) and (YJ201122).

\section{REFERENCES}

[1] Ergun, Gulsen;Somer, Tarik G; Kisakurek; Bilgin. ANALYSIS OF DRYING RATE MECHANISM IN COMPLEX SOLIDS[C].Soc of Chemical Engineers, Japan, Tokyo,pp:127-132, April 1984.

[2] Gao Jianjun ,Guo yuhua, Zhou hemin, Wang haifeng. Modeling and Calculation of parameters of coal moisture.Fuels and Chemicals, vol.43,pp:1-7, May 2012.

[3] Passino K M. Biomimicry of bacterial foraging for distributed optimization and control.IEEE Control Systrms Magazine, vol.22,pp:52-67,March 2002.

[4] Dasgupta S, Biswa A, Abraham A. et al. Adaptive computational chemotaxis in bacterial foraging optimization: An analysis. IEEE Transactions on Evolutionary Computation, vol.13,pp:919-941, April 2009.

[5] Hu Jie. Study on improvement and application of BFO algorism. [D]. Wuhan: Wuhan University of Science and Technology, pp:29-31, 2012. 